\title{
Ontogenia e aspectos comportamentais da larva de Phasmahyla guttata (Lutz, 1924) (Amphibia, Anura, Hylidae)
}

\author{
Paulo Nogueira da Costa ${ }^{1,3}$ \& Ana Maria Paulino Telles de Carvalho e Silva ${ }^{2}$ \\ ${ }^{1}$ Departamento de Zoologia, Universidade Federal do Rio de Janeiro - UFRJ, \\ Cidade Universitária, CP 68.044, CEP 21944-970, Rio de Janeiro, RJ, Brasil \\ ${ }^{2}$ Departamento de Zoologia, Universidade Federal do Estado do Rio de Janeiro - UFRJ, \\ CEP 22290-240, Rio de Janeiro, RJ, Brasil, e-mail: atelles@unirio.br \\ ${ }^{3}$ Autor para correspondência: Paulo Nogueira da Costa, \\ e-mail:nogpj@yahoo.com.br, http://www.ipub.ufrj.br
}

COSTA, P.N. \& CARVALHO-E-SILVA, A.M.T. Ontogeny and behavioral aspects of the larva of Phasmahyla guttata (Lutz, 1924) (Amphibia, Anura, Hylidae). Biota Neotrop., 8(4): http://www.biotaneotropica.org.br/ v8n4/en/abstract?short-communication+bn01508042008

\begin{abstract}
The genus Phasmahyla Cruz, 1990 is composed of small anurans, whose spawns are deposited on leaves above forested streams. Their tadpoles are neustonic and presents dorsal buccal funnel. In the city of Rio de Janeiro the genus is represented by P.guttata (Lutz 1924). Tadpoles of this species were collected in the Rio dos Macacos, Horto Florestal, Rio de Janeiro and their ontogenetical development were studied. Larvae hatch in the stage 25 (ca $16.5 \mathrm{~mm}$ ), remaining in this stage until an average length of $18.4 \mathrm{~mm}$. The buccal funnel grows from $1.9 \mathrm{~mm}$ (stage 25) to $7.1 \mathrm{~mm}$ (stage 40), been completely absorbed in the end of stage 42 . The tail grows gradually until stage 41 , when the tadpoles reach theirs biggest size (average length of $55.1 \mathrm{~mm}$ ). In stage 41, tail start to be absorbed, disappearing completely in the stage 46. In the streams, the tadpoles are distributed in schools, constituted by tadpoles of several development stages. The composition of schools changed throughout the year. In the months of June and July the occurrence of newly hatched tadpoles was not observed, with the appearance of the first newly hatched in the month of August. A greater number of newly hatched tadpoles were observed in March, April and May. Five spawns were collected, all deposited in nests of leaves rolled into funnel shape. To make up the nests, the pair used only fresh leaves, which are longer than wide.
\end{abstract}

Keyworks: Phyllomedusinae, spawning, tadpole, development.

COSTA, P.N. \& CARVALHO-E-SILVA, A.M.T. Ontogenia e aspectos comportamentais da larva de Phasmahyla guttata (Lutz, 1924) (Amphibia, Anura, Hylidae). Biota Neotrop., 8(4): http://www.biotaneotropica.org.br/v8n4/ pt/abstract?short-communication+bn01508042008

Resumo: O gênero Phasmahyla Cruz, 1990 é composto por pequenos anuros, cujas desovas são depositadas em folhas acima de córregos na mata. Seus girinos são neustônicos e apresentam funil bucal dorsal. Na cidade do Rio de Janeiro o gênero está representado por P.guttata (Lutz 1924). Girinos desta espécie foram coletados no Rio dos Macacos, Horto Florestal, Rio de Janeiro e sua ontogenia foi estudada. A larva eclode no estágio 25 (com cerca de $16,5 \mathrm{~mm}$ ), permanecendo neste estágio até um comprimento médio de 18,4 mm. O funil bucal cresce de $1,9 \mathrm{~mm}$ (estágio 25) a 7,1 $\mathrm{mm}$ (estádio 40), sendo completamente absorvido no final do estágio 42. A cauda cresce gradualmente até o estágio 41, quando os girinos alcançam as maiores dimensões (comprimento médio de 55,1 mm). No estágio 41, a cauda começa a ser absorvida, desaparecendo completamente no estágio 46. Nos córregos, os girinos são distribuídos em cardumes, compostos por indivíduos de diferentes estágios de desenvolvimento. A composição dos cardumes variou ao longo do ano. Nos meses de junho e julho a ocorrência de girinos recém-eclodidos não foi observada, com o aparecimento dos primeiros recém-eclodidos em agosto. Um maior número de girinos recém-eclodidos foi observado em março, abril e maio. Cinco desovas foram coletadas, todas depositadas em ninhos de folhas enroladas em forma de funil. Para a confecção dos ninhos, o casal usou apenas folhas vivas, mais longas do que largas.

Palavras-chave: Phyllomedusinae, desova, girino, desenvolvimento. 


\section{Introdução}

As espécies da subfamília Phyllomedusinae Günther, 1858, distinguem-se dos demais hilídeos por apresentarem pupila vertical, locomoção em marcha, coloração verde, desova acima da superfície da água e girinos com espiráculo ventral (Cruz 1982). Esta subfamília distribui-se na América latina, do México à Argentina, e encontra-se dividida atualmente em sete gêneros: Agalychnis Cope, 1864, Cruziohyla Faivovich et al. 2005, Hylomantis Peters 1873, Pachymedusa Duellman 1968, Phasmahyla Cruz 1991, Phrynomedusa Miranda-Ribeiro 1923 e Phyllomedusa Wagler 1830. No Brasil são encontradas 36 espécies distribuídas em cinco gêneros (Frost 2008).

O gênero Phasmahyla Cruz, 1990, é composto por animais de pequeno porte (comprimento rostro-anal de 29,0 a 45,5 mm) com saco vocal e fendas vocais ausentes, que desovam em ninhos de folhas enroladas e possuem girinos com funil bucal dorsal (Cruz 1990). Este gênero encontra-se distribuído na região da Mata Atlântica do sul da Bahia ao norte do Paraná e possui quatro espécies descritas: P. guttata (Lutz 1924), encontrada no litoral dos Estados do Rio de Janeiro, São Paulo e norte do Paraná; P. jandaia (Bokermann \& Sazima 1978), encontrada em Minas Gerais; P. cochranae (Bokermann 1966), que ocorre no norte do Estado de São Paulo, sul do Estado de Minas Gerais e sudoeste do Estado do Rio de Janeiro e P. exilis (Cruz 1980), que ocorre no sul do Estado da Bahia e no Estado do Espírito Santo (IUCN 2008).

Izecksohn \& Carvalho-e-Silva (2001) abordam alguns aspectos do comportamento do girino, da vocalização e da reprodução de Phasmahyla guttata. Citam também, que essa espécie ocorre na Mata Atlântica da Serra do Mar e sofreu um declínio populacional nas últimas décadas. Como a maioria dos anfíbios possui forma larval aquática e adultos terrestres os processos seletivos que agem sobre as larvas e adultos podem agir isoladamente causando a divergência entre larvas de espécies afins e a convergência entre larvas de espécies afastadas (Dunn 1924). Estudos ontogenéticos em anuros podem contribuir para o esclarecimento das relações filogenéticas destes animais, constituindo uma importante ferramenta para elucidar questões taxonômicas entre os grupos. No presente trabalho é descrito o desenvolvimento do girino, aspectos da formação de cardume e a desova de Phasmahyla guttata.

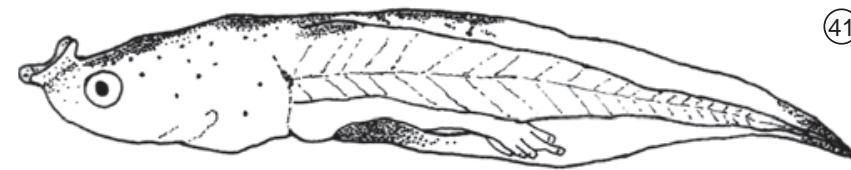

$10 \mathrm{~mm}$

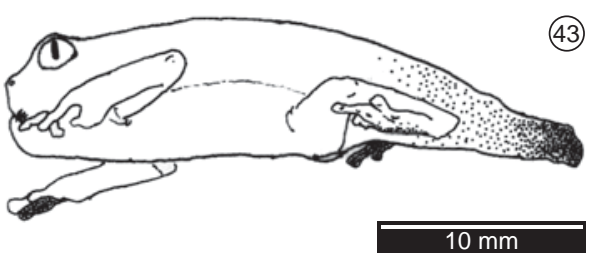

(43)

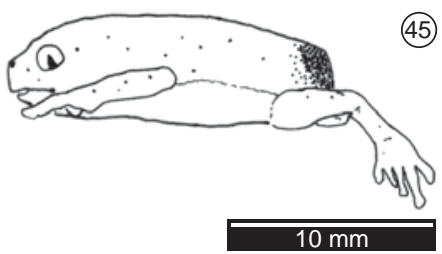

(5)

Figura 1. Vista lateral dos girinos de Phasmahyla guttata nos estágios 41, 42, 44, 45 e 46.
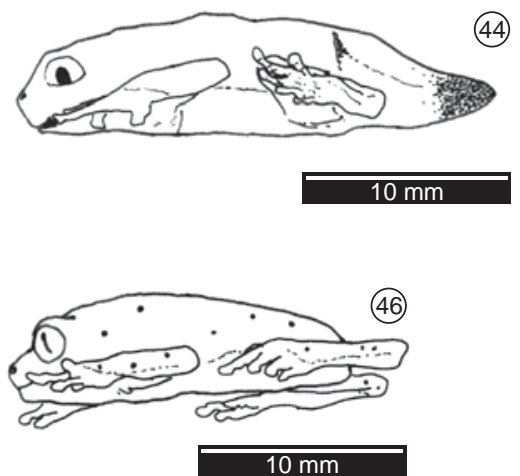

Figure 1. Lateral view of the Phasmahyla guttata tadpoles in the stages 41, 42, 44, 45 and 46. 
depositado na coleção do Laboratório de Biossistemática de Anfíbios (LABAN) da Universidade Federal do Estado do Rio de Janeiro (UNIRIO) (lotes 3191, 3192, 3193, 3194, 2731).

Os esquemas do desenvolvimento dos girinos e dos recém metamorfoseados foram realizados sob microscópio estereoscópico acoplado a uma câmara clara, no Laboratório Integrado de Microscopia e Análise de Imagens (LIMAI) da UNIRIO. A descrição da coloração dos animais foi realizada através do catálogo de cores para naturalistas de Smithe (1975).

Os cardumes foram observados em horários variados do dia, entre julho de 2004 e dezembro de 2005, por períodos de tempo de $30 \mathrm{mi}-$ nutos a duas horas. Durante as observações foi anotado o número de indivíduos nos cardumes, a composição dos cardumes, a direção de deslocamento dos girinos e a distância entre eles. A distância entre os girinos nos cardumes foi medida no ambiente de coleta com o auxílio de uma régua. Todos os indivíduos dos cardumes foram capturados com um puçá e contados. Indivíduos de tamanhos muito diferentes foram coletados e levados ao laboratório para a determinação do estágio de desenvolvimento.

\section{Resultados}

Os girinos eclodem no estágio 25 com cerca de $16,5 \mathrm{~mm}$ e permanecem neste estágio até um comprimento médio de $18,4 \mathrm{~mm}$. O maior girino no estágio 25 tinha $24,2 \mathrm{~mm}$ de comprimento total.

O funil bucal cresce de um comprimento médio de $1,9 \mathrm{~mm}$, no estágio 25, até alcançar um tamanho máximo médio de 7,1 mm, no estágio 40. A partir deste estágio o funil bucal começa a ser absorvido desaparecendo por completo no final do estágio 42. Neste estágio foram observadas as maiores variações no comprimento do funil bucal, de um comprimento máximo de 5,9 $\mathrm{mm}$ até seu desaparecimento, dando origem a uma boca de 3,9 mm (Figura 1). A cauda cresce de forma progressiva até o estágio 41, quando o girino alcança seu maior tamanho com um comprimento médio de 55,1 mm. A partir do estágio 41 a cauda começa a regredir, desaparecendo por completo no estágio 46 (Figura 1). No estágio 43 ocorre a maior variação do comprimento total com um comprimento máximo de 48,9 mm e mínimo de $32,7 \mathrm{~mm}$, re etindo as variações sofridas no comprimento da cauda (Figuras 1 e 2). O corpo cresce rapidamente do estágio 25 ao 27, dobrando seu tamanho. A partir do estágio 27 o crescimento corpóreo é mais lento e contínuo, o corpo continua crescendo até a idade adulta (Figura 2).

À medida que se aproxima da metamorfose (estágio 41), a região rostral do girino começa a tomar as características do adulto (Figuras 3 a 5). A distância interorbital cresce do estágio 25 ao estágio 39 e após este estágio começa a diminuir. A distância internasal cresce até o estágio 38, decrescendo de acordo com a proximidade da metamorfose. A distância entre o olho e a narina cresce até o estágio 30 e permanece quase que constante após este estágio (Figuras 4 e 5). No animal adulto as narinas e os olhos ficam mais próximos do focinho. A distância entre o olho e o focinho diminui acentuadamente assim que o animal sai da água, no estágio 42 (Figura 3). O comprimento da mão é maior que do antebraço no estágio 42. A partir do estágio 44 a mão passa a crescer de forma menos acelerada, com suas medidas igualando-se as do antebraço no estágio 45 . No estágio 46 o comprimento do antebraço já é maior que o comprimento da mão (Figura 1).

O recém metamorfoseado mede em média 19,3 mm de CRC.

Os cardumes de $P$. guttata são compostos por indivíduos de estágios variados, sendo muitas vezes observados cardumes com girinos nos estágios 26 e 42. Os girinos são neustônicos e permanecem na superfície da água movimentando a ponta da cauda em um movimento agelar, geralmente com o funil bucal em contato com a superfície da

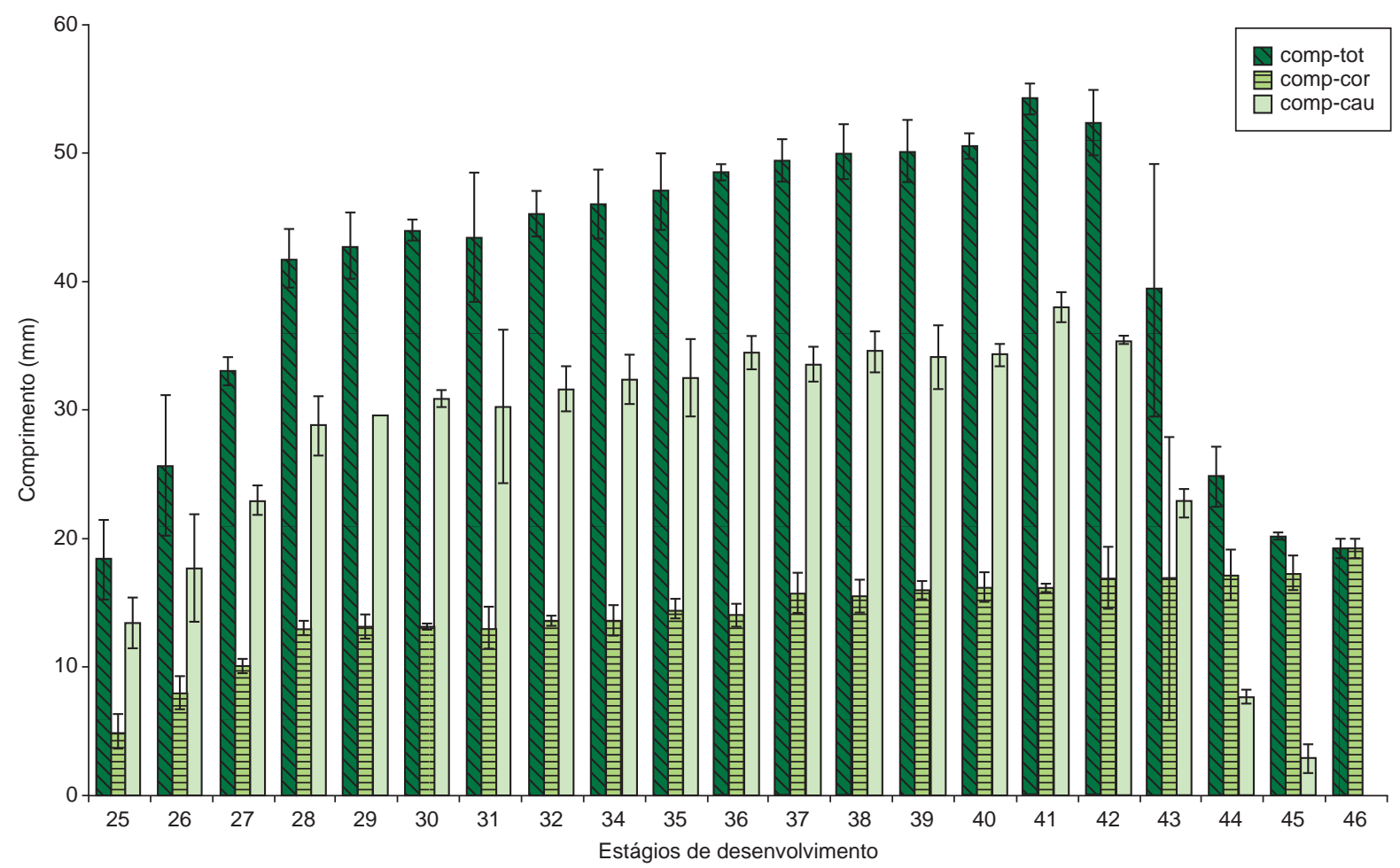

Figura 2. Valores médios de Comprimento total (Comp-tot), Comprimento do corpo (Comp- cor) e Comprimento da cauda (Comp-cau) dos girinos de P. guttata nos estágios 25 ao 46.

Figure 2. Mean values for total length (Comp-tot), body length (Comp-cor) and tail length (Comp-cau) of tadpoles of P. guttata in stages 25 to 46. 
água. Nos meses de junho e julho não foi observada a ocorrência de girinos recém eclodidos (com 16,5 mm), os quais foram registrados de março a maio e em agosto. Girinos de estágios mais avançados foram observados o ano todo.

Os cardumes são bem organizados e podem variar de 15 a 30 indivíduos, com os girinos nadando sempre voltados para a mesma direção. Os girinos mantêm uma distância regular entre si, de cerca de 30 a $40 \mathrm{~mm}$. Muitas vezes foram observados cardumes isolados formados apenas por girinos no estágio 25. Esses cardumes não se misturavam e devido ao pequeno número de girinos, homogeneidade de tamanho e de estágio de desenvolvimento entre eles, tais cardumes possivelmente são formados por indivíduos de uma mesma desova.

Foram coletadas cinco desovas (Tabela 1), todas depositadas em ninhos de folhas enroladas em formato de funil, quatro delas pendentes sobre a água e uma pendente sobre uma rocha na margem do riacho. Duas desovas estavam depositadas na face adaxial e três na face abaxial das folhas. Para a confecção dos ninhos (Figura 6), o casal usou apenas folhas vivas (mais compridas do que largas), localizadas a uma altura de até $163 \mathrm{~cm}$ da superfície da água. Duas desovas foram encontradas abertas e não se desenvolveram, as desovas encontradas $\mathrm{m}$ folhas enroladas se desenvolveram normalmente.

Uma desova foi mantida em cativeiro até sua eclosão. O último girino eclodiu 12 horas após a eclosão do primeiro. Os girinos eclodiram com cerca de $16,5 \mathrm{~mm}$ (Figura 6). Os ovos apresentavam coloração creme e estavam dispostos de forma aleatória formando uma massa compacta no interior da folha, sendo que em algumas desovas foram encontrados ovos em diferentes estágios de desenvolvimento. Os primeiros girinos a eclodirem saíram pelo fundo do funil. A eclosão da cápsula gelatinosa pelos girinos libera água, que vai aos poucos abrindo a desova, facilitando a saída dos demais girinos.

\section{Discussão}

Em alguns anuros o membro anterior esquerdo sai pela abertura do espiráculo (Duellman \& Trueb 1996), mas isso não é possível para os Phyllomedusinae devido a seu espiráculo ventral. Os membros anteriores de $P$. guttata só aparecem quando a membrana opercular se rompe.

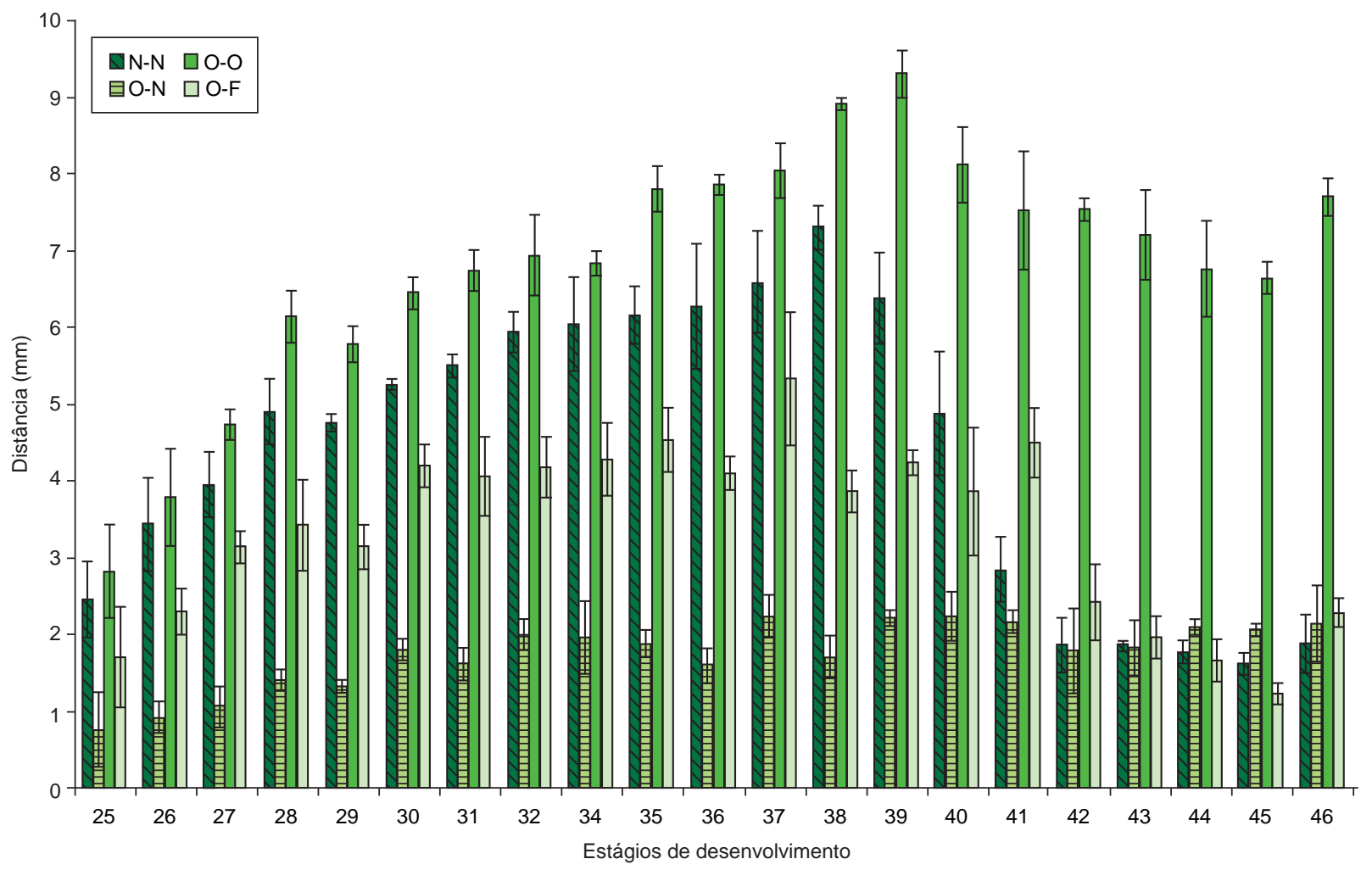

Figura 3. Valores médios de Distância internasal (N-N), Distância interorbital (O-O), Distância entre o olho e a narina (O-N) e entre olho e o focinho (O-F), nos estágios 25 a 46 dos girinos de P.guttata.

Figure 3. Median of Internasal distance (N-N), Interorbital distance (O-O), Distance between eye and nostril (O-N) and between eye and snout (O-F), in stages 25 to 46 of $P$. guttata tadpoles.

Tabela 1. Número de ovos e altura do sítio de desova de P. guttata no Rio dos Macacos, Rio de Janeiro.

Table 1. Egg number and high of egg clutch site of $P$. guttata in Rio dos Macacos, Rio de Janeiro.

\begin{tabular}{lccccc}
\hline \multicolumn{1}{c}{ Desovas egg clutch } & $\mathbf{1}$ & $\mathbf{2}$ & $\mathbf{3}$ & $\mathbf{4}$ & $\mathbf{5}$ \\
\hline Data de coleta date of collection & $10 / 2004$ & $05 / 2005$ & $05 / 2005$ & $09 / 2005$ & $05 / 2006$ \\
$\mathrm{~N}^{\text {o }}$ de ovos number of eggs & 68 & 25 & 21 & 51 & 47 \\
Distância da água em cm distance from the water in cm & 155 & 103 & 163 & 98 & 110 \\
\hline
\end{tabular}



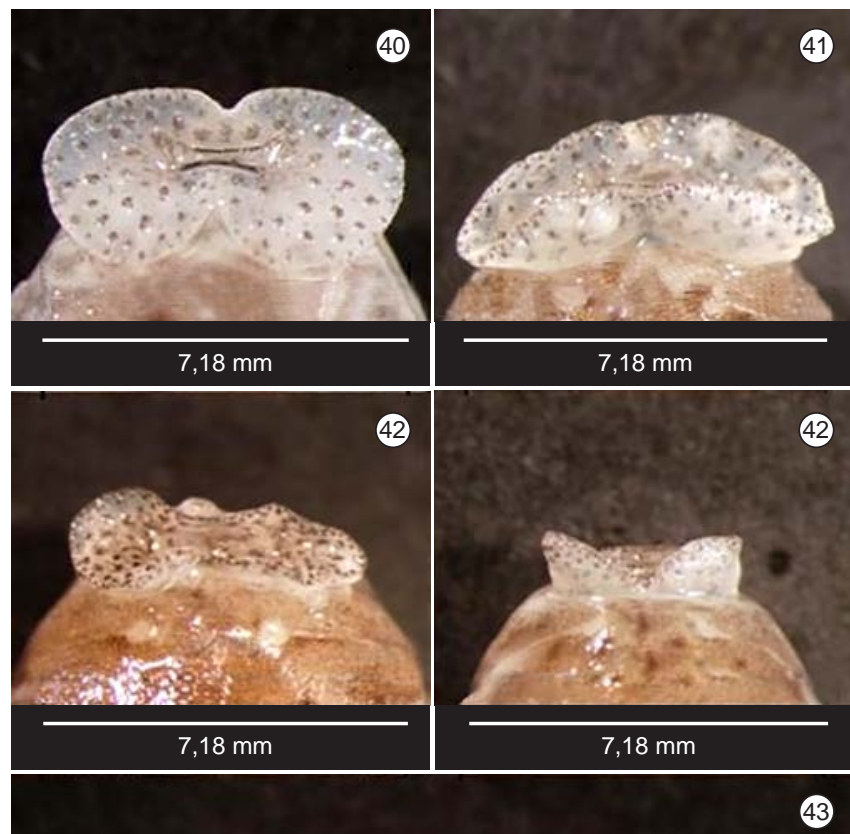

43

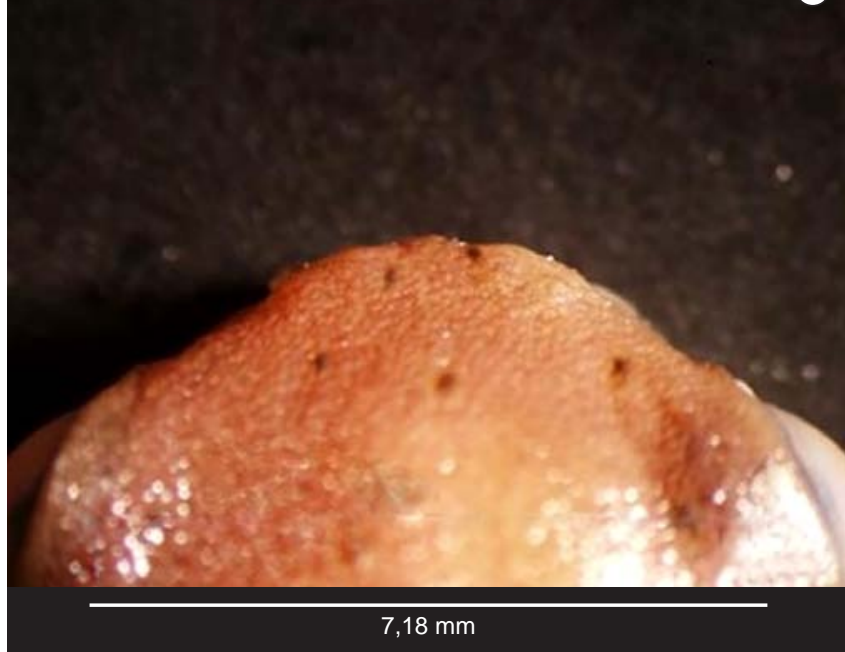

Figura 4. Vista dorsal das várias etapas de regressão do funil bucal nos estágios 40 a 43

Figure 4. Dorsal view of the various stages of regression of the buccal funnel in the stages 40 to 43 .

O único girino de $P$. guttata no estágio 37 observado por Cruz (1982), apresenta dimensões menores do que a média e igual ao menor tamanho registrado nos exemplares presentemente estudados. O girino de $P$. guttata apresenta o maior comprimento total entre os girinos de Phasmahyla conhecidos: $P$. cochranae, comprimento total de 43,0 $\mathrm{mm}$ e comprimento do corpo de $16 \mathrm{~mm}$ no estágio 36; $P$. jandaia comprimento total de $42,5 \mathrm{~mm}$ e comprimento do corpo de $14 \mathrm{~mm}$ no estágio 37; $P$. exilis comprimento total de $39,5 \mathrm{~mm}$ e comprimento do corpo de $13 \mathrm{~mm}$ no estágio 37 (Cruz 1982). O menor girino encontrado por nós no estágio 36 possuía $47,6 \mathrm{~mm}$ de comprimento total, com $13 \mathrm{~mm}$ de comprimento do corpo. Já no estágio 37 , o menor tamanho encontrado foi $47,5 \mathrm{~mm}$ de comprimento total, com $13 \mathrm{~mm}$ de comprimento do corpo. Assim, os girinos de $P$. guttata são os maiores do gênero, sendo que sua cauda longa proporciona seu maior comprimento, pois o comprimento corpo é menor que o de $P$. jandaia e de $P$. cochranae e igual ao de $P$. exilis.

Os girinos criados em laboratório cresceram menos do que os encontrados no riacho, por isso seu desenvolvimento não foi estuda-
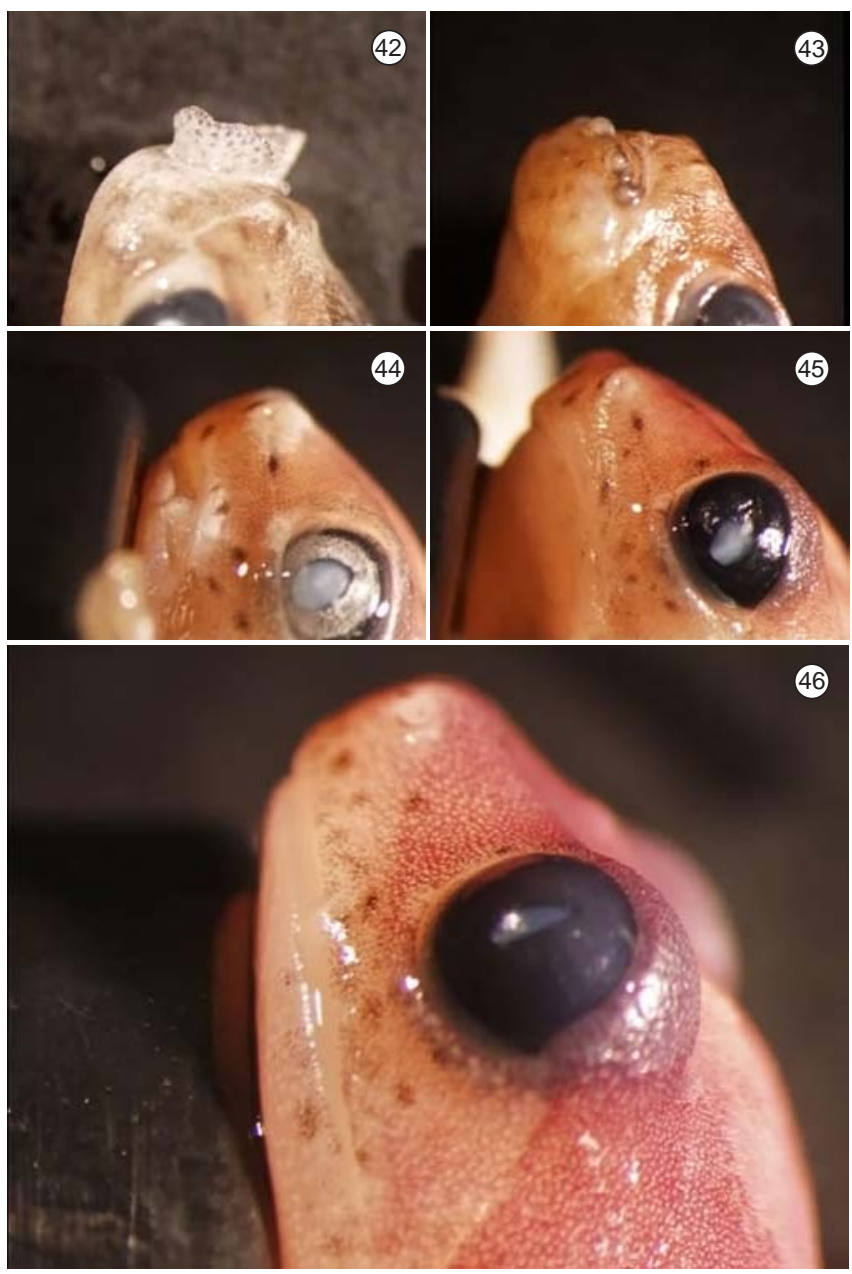

Figura 5. Vista lateral do desenvolvimento da região rostral, entre os estágios 42 a 46.

Figure 5. Lateral view of the rostral portion development, between stages 42 to 46.

do. Fatores como temperatura da água, disponibilidade e qualidade do alimento podem estar relacionados a este fato. De acordo com Caramaschi \& Jim (1983) variações nas dimensões corporais dos girinos ocorrem mesmo dentro de cada estágio e podem estar relacionadas ao crescimento do girino antes que mude de estágio ou a maior eficiência individual na tomada e na conversão de alimento.

Os girinos são classificados como neustônicos (Altig \& Mcdiarmid, 1999) e, segundo Lutz (1954), os girinos de P. guttata são gregários.

Todos os Phyllomedusinae depositam seus ovos fora do ambiente aquático, entretanto nem todas as espécies usam folhas como ninho. Phrynomedusa fimbriata Miranda-Ribeiro 1923 e Phrynomedusa marginata (Izecksohn \& Cruz, 1976), por exemplo, desovam em rochas ou troncos caídos sobre a superfície da água (Cruz 1990). Foram encontradas desovas depositadas tanto na face abaxial quanto na adaxial das folhas, diferente do observado por Bokermann \& Sazima (1978) para P. jandaia, que deposita seus ovos na face abaxial das folhas, e por nós para a população de $P$. guttata em Mangaratiba, que desova na face adaxial das folhas. De acordo com Prado et al. (2000) o tamanho das fêmeas pode estar positivamente relacionado com o número de ovos produzidos, o que pode explicar a diferença na quantidade de ovos nas desovas observadas de $P$. guttata. 

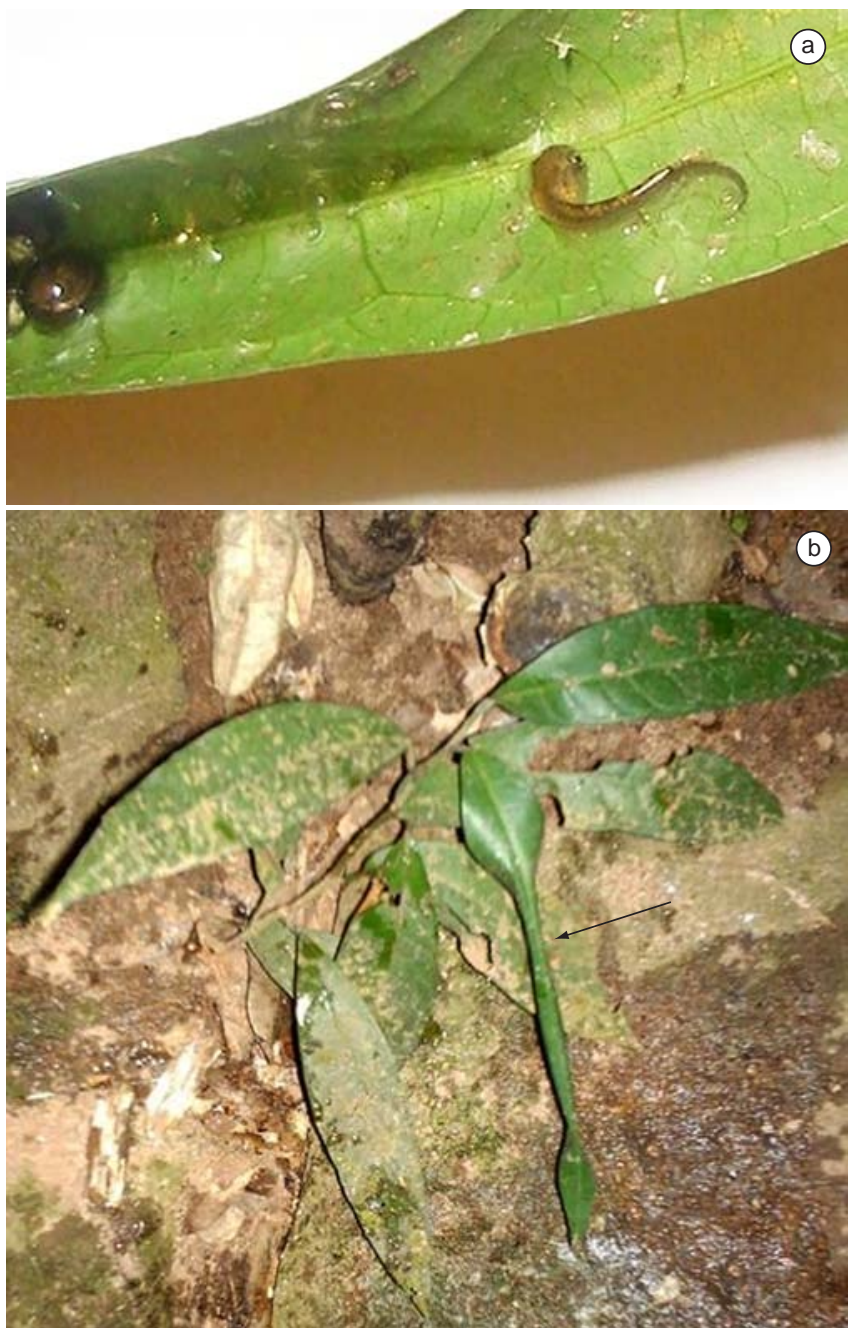

Figura 6. a) Desova de P.guttata com girino recém eclodido e b) A seta indica uma folha enrolada, onde a desova de P.guttata é depositada.

Figure 6. a) Egg clutch of P.guttata with newly hatched tadpoles and b) The arrow indicates coiled leaves, rolled into funnel shape, where the egg clutch of P.guttata is deposited.

Pyburn (1980) mostrou que as folhas enroladas fornecem proteção contra dessecação para os embriões de Phyllomedusa hypocondrialis (Daudin, 1800) durante o desenvolvimento fora da água, o que pode explicar a perda das desovas encontradas em folhas abertas.

Phasmahyla guttata, como as outras espécies do gênero, desova em folhas pendentes sobre a água. Produzem cerca de 20 a 70 ovos de cor creme, tanto na face abaxial quanto na adaxial, de folhas vivas mais compridas do que largas, localizadas a até cerca de $160 \mathrm{~cm}$ de altura da superfície da água. Os girinos são neustonicos (Altig \& Mcdiarmid, 1999) e vivem em cardumes com girinos de diferentes tamanhos e estágios de desenvolvimento.

\section{Referências Bibliográficas}

ALTIG, R. \& MCDIARMID, R.W. 1999. Body plan: development and morphology. In Tadpoles: The Biology of Anuran Larvae (McDiarmid, R.W. \& Altig, R. eds.). The University of Chicago Press, Chicago and London, p. 24-51.

BOKERMANN, W.C.A \& SAZIMA, I. 1978. Anfíbios da Serra do Cipó, Minas Gerais, Brasil. 4: Descrição de Phyllomedusa jandaia sp. n. Rev. Brasil. Biol. = Braz. J. Biol. 38(4):927-930.

CARAMASCHI, U. \& JIM, J. 1983. Observações sobre hábitos e desenvolvimento dos girinos de Phyllomedusa vaillanti Boulernger (Amphibia, Anura, Hylidae). Rev. Bras. Biol. = Braz. J. Biol. 43(3):261-268.

CRUZ, C.A.G. 1980. Descrição de uma nova espécie de Phyllomedusinae do Estado do Espírito Santo, Brasil. Rev. Bras. Biol. = Braz. J. Biol. 40(4):683-687.

CRUZ, C.A.G. 1982. Conceituação de grupos de espécies de Phyllomedusinae brasileiras com base em caracteres larvários (Amphibia, Anura, Hylidae). Arq. Univ. Fed. Rur. Rio de Janeiro 5(2):149- 171.

CRUZ, C.A.G. 1990. Sobre as relações intergenéricas de Phyllomedusinae na Floresta Atlântica (Amphibia, Anura, Hylidae). Rev. Bras. Biol. = Braz. J. Biol. 50(3):709-726.

DUELLMAN, W.E. 1970. The Hylid Frog of Middle America. Vol. 1. Monograph Museum of Natural History, University of Kansas, Kansas, p. 1-427.

DUELLMAN, W.E. \& TRUEB, L. 1996. Biology of amphibians. MacGrawKill Publishing Company, New York.

DUNN, E.R. 1924. Some Panamanian frogs. Occ. Pap. Mus. Zool. Univ. Mich. 151:1-12.

FROST, D.R. 2008. Aphibian Species of the World: an online reference. http:// research.amnh.org/herpetology/amphibia/index.html (último acesso em 15 julho 2002)

IUCN. Conservation International, and NatureServe. 2006. Global Amphibian Assessment (GAA). <www.globalamphibians.org>. (último acesso em 17 maio 2008).

GOSNER, K.L. 1960. A simplified table for estaging anuram embryos and larvae with notes on identification. Herpetologica 16:183-190.

IZECKSOHN, E. \& CARVALHO E SILVA, S.P. 2001 Anfíbios do Município do Rio de Janeiro. Ed. UFRJ, Rio de Janeiro.

LUTZ, A. 1924. Sur les Rainettes des environs de Rio de Janeiro. C. R. Soc. Biol. Paris 90:241.

LUTZ, B. 1954. Anfíbios anuros do Distrito Federal. Mem. Inst. Oswaldo Cruz 52:155-197.

PRADO, C.P.A., UETANABARO, M. \& LOPES, F.S. 2000. Reproductive strategies of Leptodactylus chaquensis and Leptodactylus podicipinus in the Pantanal, Brazil. J. Herpetol. 34(1):135-139.

PYBURN, W.F. 1980. The function of eggless capsules and leaf in the nests of the frog Phyllomedusa hypocondrialis (Anura, Hylidae). Proc. Biol. Soc. Wash. 93(1):153-167.

SMITHE, F.B. 1975. Naturalist's Color Guide. American Museum of Natural History, New York.
Recebido em 22/09/08

Versão reformulada recebida em 11/11/08

Publicado em 12/11/08 